\title{
Génétique de la sclérose en plaques
}

La sclérose en plaques est une maladie comportant une composante génétique et une composante liée à l'environnement. L'un des gènes de susceptibilité à l'affection serait lié au locus du complexe majeur d'histocompatibilité.

Joël Oger

Associate professor à l'université de Colombie-Britannique.

\section{Olivier Sabouraud}

Professeur de neurologie, chef de service de neurologie à l'université de Rennes.

\section{Barry Arnason}

Professeur de neurologie, chef $d u$ département de neurologie à l'université de Chicago.

\section{ADRESSES}

J. Oger : Health Sciences Center Hospital, 2211 Wesbrook Mall, Vancouver, BC, V6T 1W5, Canada.

O. Sabouraud : Hôpital Pontchaillon, rue Henri Le Guillou, 35033 Rennes Cedex, France.

B.G. Arnason : université de Chicago, 950 E 59th street, Chicago, Illinois 60637 USA.

$m / s n^{\circ} 1$ vol. 3, janvier 87 a sclérose en plaques (SEP) est une maladie démyélinisante inflammatoire de diagnostic souvent difficile et d'étiologie inconnue. L'ensemble des études que nous allons discuter plus loin repose sur la prévalence de la SEP. La prévalence est établie à partir du nombre de cas où la maladie est " cliniquement définie ", c'est-à-dire des cas où le diagnostic est établi avec certitude. Il est certain que cette restriction retarde le moment du diagnostic [1] et réduit la fréquence de la maladie de façon considérable. Ainsi, par exemple, von Gorgi, en Suisse, a montré que près de $20 \%$ des cas de SEP relevés lors d'autopsies systématiques étaient cliniquement silencieux. Dans une étude sur la population de la Colombie-Britannique, 2596 cas de SEP "définie ou probable" ont été relevés par Sweeney mais 1508 cas de "SEP possible " ont aussi été dénombrés. Étudiant deux cents de ces cas de "SEP possible " par Résonance Magnétique (IRM), Paty a retrouvé des lésions typiques chez près de
$70 \%$ des malades et chez plus de $50 \%$ des cas de névrite optique cliniquement isolée [3]. L'impression qui ressort est donc que $40 \%$ à $50 \%$ des malades porteurs de lésions de SEP à un moment donné ne sont pas reconnus cliniquement et sont donc exclus des études épidémiologiques que nous allons discuter plus loin.

\section{Répartition géographique}

La SEP est plus fréquente dans le nord et le centre de l'Europe, a u Canada et dans le nord des États-Unis (prévalence 40-100/ $100000)$ : parmi les individus âgés de 20 à 50 ans, une personne sur 500 a la maladie (figure 1). Dans ces régions de haute prévalence, certains groupes ne sont cependant pas atteints par la SEP. La maladie est inexistante chez les Indiens d'Amérique et rare chez les Américains d'origine japonaise ou chinoise $(6,6 / 100000)$; en 1982 , deux cas seulement de SEP avaient été enregistrés chez les gitans de Hongrie. Chez les Noirs américains, la prévalence de la SEP est à peu près deux fois 


\section{RÉFÉRENCES}

1. Izquierdo G, Hauw JJ, Lyon-Caen $\mathrm{O}$, et al. Value of multiple sclerosis diagnostic criteria 70 autopsy - confirmed cases. Arch Neurol $1985 ; 42: 848-50$.

2. Sweeney VP, Sadovnick AD, Brandejs V. Prevalance of multiple sclerosis in British Columbia. Can J Neurol Sci 1986 ; 13 : 47-51.

3. Paty DW, Hashimoto SA, Oger J, et al. Magnetic Resonance Imaging (MRI) in multiple sclerosis : a prospective evaluation of usefullness in diagnosis. Neurology (NY) $1986 ; 36$ (sup. 1) : 186.

4. Aperovitch A, Bouvier MH. Geographical pattern of death rates from multiple sclerosis in France : an analysis of 4912 deaths. Acto Neurol Scand 1982 ; 66 : 454-61.

5. Dean G. Annual incidence prevalance and mortality of multiple sclerosis in white south African born and in white immigrants to south Africa. Br Mad J (Clin Res) 1967 ; 11 : 724-30.

6. Kurtzke JF, Beebe GW, Norman JE. Epidemiology of multiple sclerosis in United States veterans III migration and the risk of multiple sclerosis. Neurology (NY) $1985 ; 35: 672-8$.

7. Kurtzke JF, Hyllested K. Multiple sclerosis on the Färö islands. Clinical and epidemiological features. Ann Neurol 1979 ; 5 : 6-21.

8. Oger J, Sabouraud O, Fauchet R, et al. Étude du système majeur d'histocompatibilité dans la sclérose en plaques. Rev Neurol (Paris) 1976 ; 132 : 89-97.

9. Marcadet A, Massart C, Semana G, et al. Association of class II HLA DQ $\beta$ chain DNA restriction fragments with multiple sclerosis. Immunogenetics 1985 ; 22 : 93-6.

10. Madigand M, Oger J, Fauchet R, et al. HLA profiles in multiple sclerosis suggest two forms of the disease and existence of protective haplotypes. J Neurol Sti $1982 ; 53$ : 519-29.

11. Stewart GJ, Basten A, Kirk RL. Strong linkage disequilibrium in MS. Tissue antigens. moindre que dans la population américaine de race blanche. La SEP est rare en Extrême-Orient (1-4/100 000), dans les régions tropicales et subtropicales de l'Amérique, et elle est inconnue chez les Noirs d'Afrique. La répartition géographique de la SEP a été interprétée de diverses manières et a amené certains auteurs à envisager le rôle d'un facteur environnemental qui n'existerait que dans ces régions. Un gradient nord-sud dans la prévalence de la SEP a aussi été retrouvé à l'intérieur même des États-Unis, de l'Australie, du Japon, et en Europe. Cela fait penser qu'un facteur lié au climat et rencontré relativement tôt dans la vie pourrait favoriser la SEP. Cependant G. Ebers a récemment suggéré qu'en fait la répartition des émigrants venant d'Europe du Nord présentait elle aussi un gradient nord-sud. En France aussi, la prévalence de la SEP présente un gradient nord-sud [4] parallèle à l'influence de l'origine nord-européenne.

Des résultats apparemment en faveur de l'hypothèse d'une étiologie infectieuse ont été obtenus par l'étude des migrations en Afrique du Sud, en Israël, en Australie et aux États-Unis. Les études venant d'Afrique du Sud et d'Israël sont sujettes à de sérieuses critiques. En Afrique du Sud, par exemple, les habitants ayant récemment immigré d'Angleterre semblent plus susceptibles de développer la SEP (prévalence $=40,3 / 100000$ ) que les Sud-Africains de souche anglaise ayant passé toute leur vie en Afrique du Sud (prévalence = $12,7 / 100000)$. Il en avait été conclu que les personnes ayant passé leur enfance en Angleterre avaient rencontré dans cet environnement un facteur de risque qui était absent ou moins fréquent en Afrique du Sud. Cette différence de prévalence est cependant extrapolée à partir d'un petit nombre de cas cliniques [5] et elle est bien moindre que la différence entre les Noirs bantous (chez qui la SEP est inconnue), et les Blancs d'Afrique du Sud. Étudiant la fré- quence de la SEP chez les anciens combattants américains, Kurtzke a confirmé que, chez ceux qui ont passé leur enfance dans le sud (prévalence basse) avant de s'installer dans le nord, la SEP est moins fréquente que chez les Américains nés dans le nord, y compris ceux qui se sont plus tard établis dans le sud [6]. Chez ces derniers, la migration semble cependant s'être accompagnée d'une réduction du risque. Malheureusement, cette seconde assertion repose encore sur une différence faible entre les cas et les témoins $(39 / 4.980$ SEP et $53 / 4.709$ témoins).

Kurtzke a attiré l'attention sur une épidémie de SEP survenue dans les îles Färö après l'occupation anglaise au cours de la Deuxième Guerre mondiale (24 cas en 18 ans pour une population de 45 000). Comme la SEP n'y avait pas été signalée auparavant, ces chercheurs postulent que les Anglais y ont introduit un agent infectieux provoquant la maladie [7]. Cette thèse suppose que l'on sache avec certitude si la SEP était vraiment inexistante dans les îles Färö avant la venue des Anglais. Il n'y a toujours pas de neurologue aux îles Färö, et les comptes rendus médicaux d'autrefois sont bien peu documentés. Il n'est pas certain qu'on puisse jamais résoudre ce problème d'autant que des foyers de SEP ont aussi été repérés en Europe continentale (par exemple, dans certaines communes des Côtes-duNord en Bretagne).

Les conclusions suivantes nous paraissent en fait justifiées : (a) La SEP est essentiellement une maladie des Européens du Nord et de leurs descendants. (b) Les personnes qui appartiennent à des populations à risque faible et qui migrent dans des régions à haut risque ne voient pas leur risque augmenter de façon substantielle. (c) L'observation de cas groupés dans le temps n'est pas prouvée de façon définitive. (d) Le fait que la SEP se déclare chez des individus d'ascendance nord européenne mais non dans les populations indigènes (Maoris de 


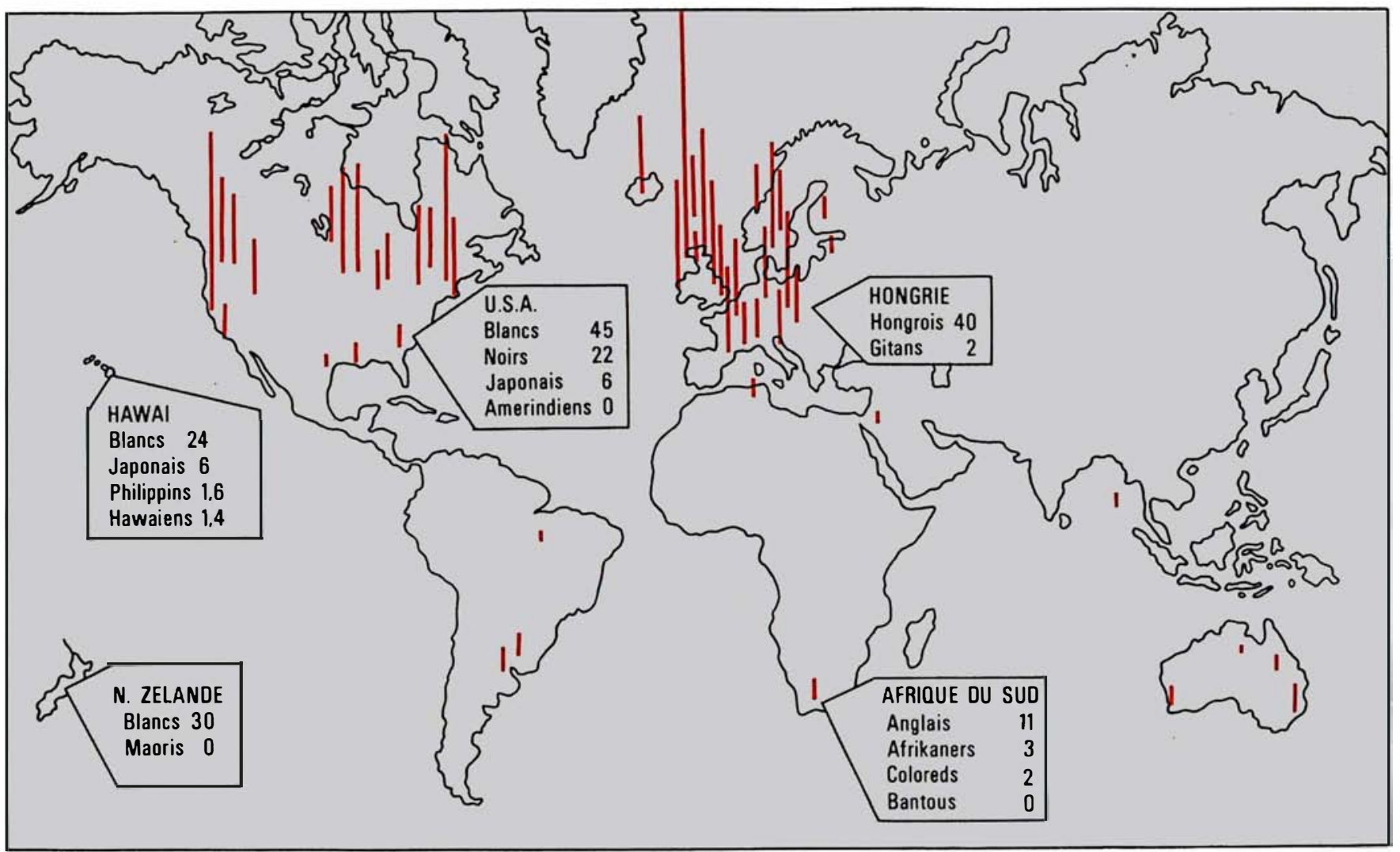

Figure 1. Répartition de la SEP dans lo monde. Chaque barre rouge verticale représente une étude épidémiologique. La taille de chaque barre est proportionnelle à la prévalence publiée. Échelle : Vancouver, prévalence 100/100 000.

Nouvelle-Zélande et Bantous d'Afrique du Sud) indique que, si des facteurs d'environnement existent, ils sont également présents dans les climats à risque bas et indique aussi que le facteur héréditaire est essentiel.

\section{Études des groupes HLA en Europe du Nord}

Il est maintenant établi que, chez les malades de race blanche, les allèles d'histocompatibilité A3, B7, DR2 et DW2 sont plus fréquents [8]. Ces données sont habituellement interprétées de la façon suivante : il existe un allèle de susceptibilité à la maladie lié au système HLA et situé plus près de $D$ que de $B$ dans la région HLA du chromosome 6. Il faut noter que l'association HLA-SEP est cependant loin d'être totale. Les études utilisant le polymorphisme des fragments de restriction de l'ADN (RFLP)* ont récemment démontré que l'haplotype DR2 est fréquemment associé à 2 fragments appelés DQR 1 et DQR2,6. Marcadet et Cohen [9], utilisant cette technique, ont montré que $84 \%$ des malades contre $55 \%$ des témoins possédaient le RFLP DQR2,6. DQR 2,6 est présent chez $97 \%$ des SEP présentant DR2 et seulement $63 \%$ des témoins.

La majorité des études révèle aussi une augmentation de B8 et DR3, Dw3. Nous avons suggéré, avec Madigand, qu'un allèle situé entre B8 et DR3, Dw3 pourrait agir en facilitant ou en amplifiant la susceptibilité à la SEP [10]. Ceci pourrait s'expliquer par la complémentation génique, phénomène mis en évidence dans d'autres processus autoimmuns. A2, B12 et DR7, Dw7 sont diminués dans la SEP. Il est possible qu'un allèle se trouvant sur l'haplotype A2-B12-DR7, Dw7 protège contre la maladie. La diminution de A2-B12-DR7, Dw7 est cependant plus évidente parmi les cas de SEP ne portant ni B7, ni DR2, Dw2. Ces résultats font penser qu'il existe deux allèles de protection dans la population témoin. Il existerait donc aussi deux gènes de susceptibilité à la SEP, un seul d'entre eux étant lié à B7-DR2, Dw2. Finalement, la SEP pourrait en fait représenter

- Voir lexique $m / s n^{\circ} 3$, vol. 2, p. 152. 
plusieurs maladies ou mécanismes pathogéniques.

D'autres marqueurs génétiques de la SEP ont été découverts : les allotypes (S et SS) de la properdine sont plus fréquents [11], de même que certains allotypes $\mathrm{Gm}$ des IgG [12]. Les allotypes $\mathrm{Gm}$ sont codés par un gène présent sur un chromosome différent du 6 , ce qui tendrait à confirmer une complémentation génique.

\section{Études dans $d^{\prime}$ autres populations}

La prévalence de la SEP chez les Noirs américains est à peu près la moitié de ce qu'elle est chez les Blancs américains, alors que la SEP est une maladie inconnue en Afrique noire. Chez les Noirs africains, les allèles B7 et DR2 sont assez fréquents, mais Dw2 est rare (tableau I). Chez les Blancs, DR2 et Dw2 sont étroitement associés, alors qu'ils ne le sont pas chez les Noirs. Les études faites sur des Noirs américains ayant une SEP ont fait apparaître une nette augmentation de Dw2, essentiellement due à la rareté de Dw2 dans la population noire témoin. Aux États-Unis, la prévalence de la SEP chez les Noirs est plus élevée dans le nord que dans le sud et ceci, comme la présence de Dw2 chez les Noirs ayant la SEP, pourrait refléter un apport plus élevé de gènes blancs chez les Noirs américains du nord.

$\mathrm{Au}$ Japon, la SEP est rare $(1-4 / 100000)$; B7 est également rare et n'est pas augmenté chez les Japonais atteints de SEP. Ces données pourraient indiquer que, dans des populations ou des groupes ethniques différents, il existe différents allèles de susceptibilité à la maladie, ou bien que, dans des populations différentes, un allèle universel de susceptibilité à la SEP est lié à des marqueurs différents. Il est bien connu que les allèles HLA en déséquilibre de liaison les uns avec les autres different d'un groupe ethnique à l'autre. Chez les Blancs, B7-DR2 et B8-DR3 sont en déséquilibre de liaison, alors que, chez les Japonais, Bw52 est lié à DR2, et DR2 est lié à Dw12.
Pour cette raison, il est possible que les allèles HLA en déséquilibre avec un gène universel de susceptibilité à la SEP soient eux aussi différents.

Dans au moins une autre population, il est clair que DR2 n'est pas lié à la SEP : la maladie est pratiquement inconnue chez les Gitans hongrois. Pourtant, parmi les Gitans non atteints, $38 \%$ sont porteurs de DR2 ; cette fréquence est l'une des plus élevées qui soient connues. Chez les Gitans, ce sont $\mathrm{B} 12$ et Bw52 (mais pas B7) qui sont en déséquilibre de liaison avec DR2 [13]. Nous en concluons que, chez les Gitans, l'allèle de susceptibilité à la SEP n'est probablement pas présent malgré la présence de DR2. A partir des résultats précédents, nous pensons qu'en général, dans les populations étudiées, la prévalence de la SEP suit la prévalence des antigènes $B 7$ et Dw2.

\section{Études familiales}

Si une maladie est génétiquement déterminée, les études familiales devraient confirmer les études faites dans des populations. Chez les proches parents (premier degré) de malades atteints de SEP, le risque de contracter la maladie est de 15 à 20 fois plus grand. Cela peut provenir du milieu de vie commun ou, au contraire, de facteurs génétiques communs comme le patrimoine HLA. Cet accroissement du risque doit être considéré à sa juste valeur : pour les enfants d'un malade atteint de SEP, le risque de contracter la SEP est de l'ordre de $1 \%$; pour les frères et sœurs d'un malade atteint, ce risque est de l'ordre de $3 \%$ [14].

Stewart et coll. ont analysé le lien qui existe entre la SEP et les haplotypes HLA chez 100 paires de frères et sœurs ayant tous les deux une SEP [15]. Ils ont montré que $37 \%$ des frères et sœurs concordants ont le même haplotype (contre $25 \%$ dû au hasard) et que $13 \%$ ne partagent aucun haplotype (contre $25 \%$ dû au hasard).

Ceci indique de façon convaincante qu'un lien existe entre HLA
18. Ebers G, Bulman D, Sadovnick population based study of the incidence of MS among twins. $N$ Engl $J$ Med (à paraître). 
Tableau I

RELATION ENTRE LA FRÉQUENCE DE LA MALADIE ET LES CARACTÉRISTIQUES HLA DE LA POPULATION CONTRÓLE DANS CINQ GROUPES ETHNIQUES

\begin{tabular}{|c|c|c|c|c|c|}
\hline \multirow[t]{3}{*}{ Groupe éthnique } & \multirow{3}{*}{$\begin{array}{l}\text { Fréquence de la maladie } \\
\text { (/105 habitant) }\end{array}$} & \multicolumn{4}{|c|}{ HLA dans la population contrôle } \\
\hline & & \multirow[b]{2}{*}{ Fréquence de $\mathrm{B} 7$} & \multirow[b]{2}{*}{ Fréquence de DR2 } & \multicolumn{2}{|c|}{$\begin{array}{l}\text { déséquilibre de } \\
\text { liaison }\end{array}$} \\
\hline & & & & B7/DR2 & DR2/Dw2 \\
\hline $\begin{array}{l}\text { Blancs } \\
\text { Europe du Nord } \\
\text { Canada } \\
\text { États-Unis Nord }\end{array}$ & élevée $(40-100)$ & élevée & élevé & + & + \\
\hline $\begin{array}{l}\text { Noirs } \\
\text { États-Unis }\end{array}$ & basse $(20-40)$ & élevée & fréquent & + & + \\
\hline Japonais & très basse $(1-4)$ & basse & fréquent & + & + \\
\hline Gitans & absent $(0)$ & basse & très élevé & - & $?$ \\
\hline Noirs américains & absent (10) & basse & bas & $?$ & $?$ \\
\hline
\end{tabular}

et SEP. Ho et coll. ont fait une analyse de ségrégation et de liaison dans 161 familles relevées dans la littérature et comportant des cas multiples. Utilisant une méthode assez complexe d'étude familiale, ces auteurs concluent [16] que la susceptibilité à la SEP est hétérogène : dans $75 \%$ des cas, la susceptibilité à la SEP serait liée à HLA. Dans les $25 \%$ restant, il n'existerait aucune liaison avec le système HLA. Il est vraisemblable que cette conclusion est exacte ; elle pourrait aussi expliquer les cas de SEP chez des frères et sœurs ayant des haplotypes différents.

\section{Études de jumeaux}

Les études de jumeaux peuvent aider à déterminer si le risque d'avoir une maladie est déterminé par le patrimoine génétique ou par le milieu. Si une maladie est génétiquement déterminée, la concordance sera plus grande chez les jumeaux monozygotes que chez les jumeaux dizygotes. D'après les publications initiales, la concordance pour la SEP chez les jumeaux monozygotes était à peu près $25 \%$, alors que chez les jumeaux dizygotes, elle était de $\mathrm{m} / \mathrm{s} n^{\circ} 1$ ool. 3, janvier 87
$8 \%$ [17]. Au Canada, les résultats d'une étude menée à partir de cas suivis dans les cliniques de la SEP ont été colligés récemment par G. Ebers [18]. Cette étude, fondée sur une population bien quadrillée, indique que la concordance chez les jumeaux monozygotes est de $30 \%$ et que chez les jumeaux hétérozygotes, elle n'est que de $2,5 \%$. Ce dernier chiffre est semblable à la fréquence chez les autres frères et sœurs $(3 \%)$ et renforce très fortement l'hypothèse génétique de la maladie. Trois paires de jumeaux monozygotes sur quatre sont cependant discordantes pour la SEP : cette constatation démontre d'une façon frappante que les facteurs génétiques seuls ne suffisent pas à provoquer la SEP. Chez les jumeaux monozygotes, la présentation clinique de la maladie est elle aussi très variable d'un jumeau à l'autre.

\section{Modèles de maladie chez I'animal}

L'encéphalomyélite allergique expérimentale (EAE) fournit un modèle de maladie ayant un grand nombre de caractéristiques de la SEP. Cette maladie aiguë monophasique est induite par immunisation des animaux avec de la protéine basique de la myéline. Bien que l'EAE nous semble représenter plus un modèle pour l'encéphalomyélite aiguë disséminée que pour la SEP, elle est intéressante car son étude immunogénétique est très avancée. Cela a été étudié avec les rats Lewis (susceptibles) et les rats BrownNorway (résistants). Les hybrides de première génération sont susceptibles (mode de transmission de type dominant). Les générations suivantes ne sont susceptibles que si leur profil d'histocompatibilité est Lewis. Il est cependant possible de provoquer la maladie dans la race résistante Brown-Norway à condition d'employer un protocole d'immunisation suffisamment énergique, associant par exemple l'adjonction de vaccin anticoquelucheux. Chez la souris, la susceptibilité à l'EAE est aussi sous le contrôle des antigènes d'histocompatibilité mais elle est influencée par un second gène. Ce gène détermine la réponse des vaisseaux cérébraux à l'administration d'histamine. Les races sensibles à l'histamine et pourvues du patrimoine indispensable $\mathrm{H}-2$ ont une susceptibilité maximale à 
l'EAE. Des formes chroniques comportant des périodes de recrudescence de la maladie expérimentale ont été mises au point ces dernières années. Elles ressemblent beaucoup plus à la SEP ; cependant, l'immunogénétique de cette forme d'EAE reste très imparfaitement élucidée.

\section{Synthèse et discussion}

On peut considérer comme un fait établi que, chez les Européens du Nord et leurs descendants, il existe un lien entre les allèles B7, DR2/Dw2 du système HLA et la susceptibilité à la SEP.

La plupart des maladies liées au complexe HLA ont des caractéristiques communes. Celles-ci incluent une évolution chronique avec rechutes, une composante inflammatoire, une tendance familiale faible, une étiologie autoimmune et un modèle de maladie animale obtenue par injection d'un autoantigène.

Les résultats des études familiales indiquent que la concordance, pour la SEP, des jumeaux monozygotes est plus grande que celle des jumeaux dizygotes, ce qui prouve l'existence d'un facteur génétique dans le déterminisme de la SEP.

Les études HLA, chez des frères et sœurs atteints de SEP, révèlent davantage d'haplotypes communs que ne le voudrait le simple hasard. Cela démontre que le patrimoine HLA est un déterminant de la susceptibilité à la SEP. En même temps, étant donné que la concordance pour la SEP chez des frères et sœurs n'est que de $3 \%$ alors que l'allèle de susceptibilité à la SEP est porté par $50 \%$ des frères et sœurs, il s'ensuit deux possibilités : ou bien il existe plusieurs allèles de susceptibilité à la SEP et ces allèles sont hérités indépendamment, ou bien l'environnement exerce une influence complémentaire. Cette dernière hypothèse paraît plus probable du fait de la faible condordance chez les jumeaux monozygotes (qui devraient hériter de tous les allèles de suscepti-
La prévalence de la SEP varie grandement à travers le monde. Dans les zones de forte prévalence, un facteur génétique et un facteur environnemental doivent être réunis. Dans ces zones, certains groupes ethniques contractent rarement la SEP et il nous semble donc probable que l'absence du gène de susceptibilité est suffisante pour prévenir la maladie. Les personnes originaires du nord de l'Europe qui ont migré dans des zones où le risque de SEP dans la population indigène est nul (Afrique du Sud, Australie, États-Unis) courent toujours le risque de contracter la SEP. Cela conduit à deux hypothèses possibles : ou le facteur environnemental préexiste là où la SEP était inconnue, ou bien alors la population immigrante l'a apporté avec elle. Dans les deux cas, l'absence de gène de susceptibilité suffit encore à empêcher le développement de la maladie dans les populations indigènes. Il existe des zones, le Japon par exemple, où la prévalence de la SEP est faible mais non nulle. La question devient alors la suivante : est-ce que l'allèle de susceptibilité à la SEP y est rare ? Est-ce que le facteur environnemental y est rare ? Ou bien est-ce la conjonction des deux ? On doit ici souligner que, dans les populations d'Extrême-Orient où la SEP est rare, la maladie a tendance à être quelque peu différente de ce qu'elle est dans les populations d'Occident. Elle y atteint plus souvent les enfants; une forme clinique de type neuromyélite optique (maladie de Devic) s'y rencontre aussi plus fréquemment. Cela pourrait indiquer qu'en Extrême-Orient la SEP est une maladie homogène, alors qu'en Occident il s'agirait d'un agrégat de situations cliniques. Dans ces conditions, il se pourrait que des gènes de susceptibilité différents soient en cause, que le facteur présomptif de déclenchement soit différent, ou encore que le mécanisme physiopathogénique aboutissant à la destruction de la myéline soit lui aussi différent. Autant d'hypothèses qui méritent d'être explorées

\section{Summary}

In their review of genetic factors in multiple sclerosis the authors suggest that genetic factors may explain the geographical distribution of MS. In population studies $M S$ is linked to HLA and the haplotypes involved in different populations may not differ. In family studies and twin studies MS appears to be inherited as a dominant character with low penetrance but linkage with HLA is less clear. This may come from difficulties in separating affected from nonaffected siblings. It is also possible that MS could be an aggregate of different pathophysiological entities.

\section{Remerciements}

Une partie des travaux originaux rapportés dans ce texte a été financée par l'Inserm, l'Association pour la recherche sur la sclérose en plaques et le Conseil de la recherche médicale du Canada. Nous remercions M.L. Miginiac-Oger pour sa traduction du texte original et ses corrections ultérieures.

TIRÉS À PART

J. Oger : Health Sciences Center Hospital, 2211 Wesbrook Mall, Vancouver, BC, V6T 1W5, Canada. 